\title{
Les attitudes devant la Loi
}

\section{Pierre Livet}

\section{OpenEdition \\ Journals}

Édition électronique

URL : http://journals.openedition.org/ress/458

DOI : $10.4000 /$ ress.458

ISSN : 1663-4446

\section{Éditeur}

Librairie Droz

\section{Édition imprimée}

Date de publication : 1 janvier 2006

Pagination : 27-35

ISBN : 2-600-00959-0

ISSN : 0048-8046

\section{Référence électronique}

Pierre Livet, «Les attitudes devant la Loi », Revue européenne des sciences sociales [En ligne], XLIV-133 | 2006, mis en ligne le 09 novembre 2009, consulté le 04 mai 2019. URL : http:// journals.openedition.org/ress/458; DOI : 10.4000/ress.458 


\section{Pierre LIVET}

\section{LES ATTITUDES DEVANT LA LOI}

\section{NOTRE ATTITUDE DEVANT LE TEXTE DE KAFKA : DEVANT LA LOI}

Le texte de Kafka pourrait s'interpréter comme un de ces contes qui nous rappelle que la Loi nous domine de très haut, que nous sommes sans force devant elle, et que cependant nous avons notre place dans le royaume qu'elle ordonne. La Loi est transcendance, mais cette transcendance condescend à se pencher jusqu'à nous, si nous trouvons la bonne attitude: révérencieuse, mais exploratrice. Mais dans un tel conte, il y aurait eu deux ou trois personnages, dont le premier aurait tenté d'entrer en force, et aurait été repoussé, le second aurait été le paysan de l'histoire, et le troisième aurait modestement passé la porte par une quelconque ruse et aurait trouvé le chemin fait pour lui. Le texte de Kafka se tient donc sur la limite instable entre deux époques: l'époque des contes, où l'on pouvait rappeler la force et la miséricorde de la Loi, époque où la loi est encore sacralisée, et l'époque désenchantée, où nous savons bien qu'il y a derrière cette porte d'autres portes, et que nous ne trouverons pas un chemin fait pour nous, mais un dédale bureaucratique, qui n'a pas plus de sens que notre propre quête de la Loi. A ceux qui croient encore en la Loi, il suggère que supposer la Loi transcendante, c'est se condamner à ne jamais trouver le sens qu'elle a pour chacun. Et chez ceux qui n'y croient plus, il réveille l'angoisse de ne pas être à la hauteur de la Loi, de ne pas pouvoir remplacer l'ordre qui tenait à sa transcendance par des organisations bricolées par les humains.

Mais nous sommes en train de prendre le texte pour une sorte d'oracle qui nous indique la réalité de notre époque. C'est adopter devant lui la même attitude que le paysan devant la Loi. C'est que la littérature, dès qu'elle vaut comme telle, se présente toujours comme un texte sacré ${ }^{1}$. Un tel texte a forcément à nous révéler des choses profondes sur notre vie et notre époque. Et les universitaires, en lettres et sciences humaines, sont dressés pour tenir les textes qu'ils étudient pour des textes sacrés. En philosophie, par exemple, il n'est guère admis que l'on relève dans les textes classiques des raisonnements douteux et des contradictions. Si on le fait, c'est pour se donner ensuite le beau rôle de celui qui a montré la cohérence plus profonde de la pensée de l'auteur. En littérature, cependant, nous n'avons pas de telles exigences de cohérence dans les raisonnements. Mais nous sommes

Ce n'est pas le cas des romans policiers ordinaires, où il n'est pas iconoclaste pour le lecteur de critiquer les arguments du détective et sa pseudo-solution. 
censés céder à la fascination pour le texte, tout comme le paysan est fasciné par cette porte qu'il n'ose franchir. En ce sens, le texte de Kafka illustre à la fois l'attitude de celui qui lit, et les modes de fascination, les sortes de pièges que lui dresse celui qui écrit - et qui, pour pouvoir écrire, doit être lui-même fasciné par ces pièges. Si nous voulons mettre au point nos idées sur les figures de la Loi de nos jours, il nous faut donc d'abord identifier ce que l'on pourrait appeler la fascination ou la tentation de la littérature, puisque tout texte qui se veut littéraire déploie cette fascination, et qu'elle peut nous masquer des réalités plus banales, mais qui échappent à la littérature.

\section{L'ATTRACTEUR LITTÉRAIRE}

C'est d'ailleurs en prenant de la distance par rapport à la manière dont le texte construitla situation, et en retournant aux situations ordinaires, que nous pouvons dégager les raisons de cette fascination. Notons les éléments qui sortent de l'ordinaire. Qu'une porte soit gardée par un gardien, qu'il faille obtenir une permission d'entrer, qu'on ne puisse entrer tout de suite, ce sont des situations usuelles. Mais bien des détails ne sont pas «normaux». Par exemple, le paysan, pour regarder ce qu'il y a derrière la porte, doit se baisser. Pourtant le gardien s'est effacé. Il ne masque donc plus l'intérieur. Le paysan est donc dans une position inférieure par rapport au gardien, et cela sans qu'on nous en donne une raison ${ }^{2}$. Puis le gardien lui raconte que devant chaque salle il y a des gardiens plus puissants que lui, et de plus en plus puissants. Il ne lui dit pas qu'il y a des policiers en grand nombre, qui pourrait se coaliser contre lui. Ce serait à chaque fois un affrontement singulier, mais de plus en plus difficile. On retrouve ici des éléments de contes. Mais surtout, ce qui est mis en place - comme dans les contes - c'est une forme de mise en abyme. Seulement, au lieu que le deuxième élément inscrit dans le premier soit plus petit que celui-ci, il est au contraire plus grand, et ainsi de suite. Une telle mise en abyme n'a aucun correspondant dans une situation ordinaire (des miroirs qui se font face dévient peu à peu la perspective mais n'agrandissent pas l'image). Une mise en abyme est évidemment un attracteur du regard. Le texte suggère donc un des pièges auquel se prend aisément notre vision. D'ordinaire les choses ne deviennent pas forcément de plus en plus difficiles à mesure qu'on avance, elles peuvent soit devenir plus difficiles ou aussi difficiles, soit devenir plus faciles. Et cette croissance exponentielle de la difficulté est une autre fascination, qui explique pourquoi le paysan renonce à entrer (on ne voit pas bien pourquoi un nez pointu et une barbe maigre et noire seraient particulièrement dissuasifs).

Le texte en profite alors pour déraper dans le conte: le paysan reste de manière improbable assis sur son tabouret des jours et des années: l'inertie du paysan trouve ainsi le moyen de se mettre en correspondance avec la croissance exponentielle des difficultés, puisque les jours se transforment en années.

Le gardien converse avec le paysan, mais c'est une conversation asymétrique. Dans une conversation normale, chacun pose des questions, reçoit des réponses,

2 Un sondage sur les images mentales provoquées par cette description a fait apparaître deux types d'images; soit l'intérieur comportent des portes en hauteur, et il faut se baisser pour en voir l'enfilade, soit il comporte au contraire des degrés qui descendent. 
reçoit des questions et répond à son tour. En particulier, on évite de reposer la même question quand on sait qu'on doit obtenir la même réponse, et si la situation a changée, on ne donne plus la même réponse. Mais ici, le dialogue est asymétrique. Quand le paysan pose des questions, il reçoit toujours la même réponse, même s'il a changé ses offres en tentant de corrompre le gardien, et quand le gardien interroge, il ne se soucie pas des réponses pour poursuivre la conversation. Le paysan doit tenter de s'ajuster au gardien, le gardien ne tente même pas de s'ajuster aux réponses du paysan. Devant une telle asymétrie et un tel refus d'ajustement, la réaction ordinaire du paysan devrait être de se mettre en colère et soit de tenter de forcer le passage soit de s'en aller. Mais il n'en fait rien.

De plus, il se fixe sur le premier gardien, sans plus penser aux autres, alors que pour une activité qui dure des années, on fait d'ordinaire des plans à long terme. Sa réaction à cette galerie exponentielle des gardiens est donc de se focaliser sur le premier, et même d'inverser la mise en abyme, en se livrant à une analyse du gardien à des échelles de plus en plus petites, jusqu'à se mettre au niveau des puces du gardien, et même au dessous d'elles, puisqu'il les implore. On retrouve aussi là un processus des rituels religieux, qui mettent un enjeu transcendant, la question du salut ou de la damnation, dans les détails infimes du rite. Cette inversion fait que par rapport à l'homme de la campagne le gardien est devenu de plus en plus grand (le point zéro, le point de retournement du plus grand au plus petit, nous est donné par le moment où Kafka décrit le gardien, à l'échelle ordinaire d'un homme, avec son nez pointu et sa barbe maigre). On ne voit d'ailleurs pas pourquoi seul le paysan montre des signes de vieillesse alors que le gardien n'en montre aucun, puisque son dos ne se courbe pas comme celui du paysan. C'est là une confirmation de la puissance du gardien, sans doute.

Et finalement gardien et paysan nous proposent à eux deux un dernier attracteur, une dernière possibilité fascinante. Le paysan voit dans la loi un universel qui s'applique à tous, et le gardien lui dit que cet universel lui était réservé, lui était singulier. Nous aimerions bien en effet, si quelque chose nous est singulier, qu'il ne se réduise pas à notre idiosyncrasie contingente, et inversement, nous souhaitons rarement partager ce qui est universel avec les autres. Nos désirs seraient donc comblés si nous disposions à nous seuls d'un universel, et c'est ce que le gardien assure au paysan: cette porte qui ouvre sur la Loi universelle, elle t'était destiné à toi seul. Mais la roche Tarpéienne est tout près du Capitole, et ce mirage d'un universel réservé à une singularité échappe au paysan au moment même où il lui est révélé qu'un tel don l'attendait. Ce mirage est aussi celui du «kairos»: l'occasion unique favorable, qui évidemment ne se révèle telle qu'une fois qu'on l'a ratée. En fait, même quand on pense l'avoir saisie, et avoir réussi, il s'agit toujours d'une illusion rétrospective. Nous sommes soumis à des aléas, et la conjugaison de nos activités et de ces aléas se révèle parfois malheureuse, parfois heureuse. Nous ne pouvons le savoir qu'après coup. Mais aucune occasion qui nous soit destinée, qui soit la seule à se prêter au parfait succès de nos activités, ne nous attend ou ne nous fuit.

On voit que Kafka a concentré dans ce texte certains des attracteurs les plus fascinants pour les désirs et la pensée humaine: la mise en abîme, son inversion, la frustration systématique des symétries de la conversation pourtant poursuivie, le mariage impossible de l'universel et du singulier, et l'illusion du kairos, de 
l'occasion favorable et unique. Mais ce ne sont que des attracteurs, ce ne sont pas des descriptions de notre situation humaine effective. C'est le problème de la littérature: elle ne peut pas nous présenter des situations «normales» fonctionnant comme à l'ordinaire, parce que ces situations, nous les vivons déjà, et que nous ne portons aucun intérêt à les revivre telles quelles. Elle nous propose donc des situations limites, qui jouent des attentes ordinaires en les réorientant vers des situations fascinantes dont les processus mêmes qui sous-tendent nos attentes ordinaires font que nous avons du mal à nous en arracher. La mise en abyme est un piège pour un regard qui recherche les objets dans la profondeur, et qui cherche des indices, des étalons de profondeur. La croissance exponentielle de la puissance est un piège pour qui s'inquiète d'une première inégalité à son désavantage. La réitération d'une réponse négative malgré la poursuite de la conversation est un piège pour celui qui cherche dans la conversation un moyen de capter la faveur d'autrui sur un point précis. L'idée d'une destinée de portée universelle qui serait réservée à notre singularité est aussi un piège, comme l'idée qu'il faudrait ne pas rater l'occasion unique qui serait faite pour nous. Ce n'est pas que la littérature nous présente seulement des illusions: puisque nous sommes sensibles à ces pièges, nos fascinations vont dicter certaines de nos conduites dans la réalité, ce qui va influer sur la réalité de notre vie sociale. La littérature se contente de présenter une densité d'attracteurs fascinants nettement supérieure à celle de la vie sociale.

On peut alors se demander pourquoi nous aimons la littérature, si elle nous présente les pièges que notre cognition et nos inquiétudes se tendent à ellesmêmes. Il y a trois réponses possibles. La première est que nous n'aimons pas la littérature, mais que tout simplement elle nous attire, puisqu'elle est fascinante. La seconde est que nous pouvons goûter dans la littérature un plaisir analogue à celui des montagnes russes et autres dispositifs de foire: ils nous donnent le vertige dans des situations où aucun danger réel n'est lié au vertige. Nous pouvons goûter ce qui nous fascine sans que cela ait un effet sur notre vie quotidienne (à part le temps passé à lire ou à regarder). La troisième est que dans la vie sociale pratique, nous devons nous adapter aux situations, nous devons résoudre les conflits et les incohérences pratiques, et donc sortir des pièges fascinants. Pour revenir sur les différents pièges du texte, nous devons savoir où nous arrêter dans la mise en abyme, à quel niveau telle croissance exponentielle va s'arrêter ou se renverser, comment changer nos offres conversationnelles pour trouver des réponses plus accueillantes, ou quand rompre la relation, comment adapter des notions générales à des cas particuliers, à quel moment lancer une action alors que nous ne savons pas l'état exact des circonstances. Mais cela demande des efforts et des prises de risque. C'est plus inquiétant que le piège fascinant, puisque les conséquences du choix de tel ajustement sont bien réelles, et que nous pourrons toujours penser que nous aurions pu mieux nous adapter. La littérature nous offre les attracteurs limites, mais elle nous délivre de l'effort harassant de devoir sans cesse nous ajuster à de nouvelles irrégularités et imprévus. Rester fasciné devant l'attracteur, sans avoir à rien faire pour le modifier et pour ajuster notre conduite, est plus attirant que devoir se démener sans repères bien précis dans une situation concrète. 


\section{AUTRES ATTITUDES DEVANT LA LOI}

Mais cette tendance à rester devant la Loi au lieu de s'activer dans un espace de lois, voire entre les lois, nous semble surtout propre à des conceptions de la Loi qui ont eu une efficace dans les sociétés passées.

Certes, il faut tenir compte de notre tendance, quand nous jetons un regard rétrospectif sur les attitudes des sociétés passées, à styliser les conduites passées, en utilisant précisément pour cela des attracteurs littéraires. Il reste de la littérature dans notre regard sur l'histoire. Une rétrospective des attitudes historiques devant la Loi demeure donc encore littéraire, en ce qu'elle met en saillance ce qui reste pour nous des attracteurs de nos descriptions des rapports aux lois et à la Loi. Rappelons donc rapidement quelques uns de ces attracteurs et les attitudes qui leur sont liées.

La première d'entre elles est de relier loi et crise. Si nous ne respectons pas la loi, pensaient les grecs, alors notre société entrera en crise. D'où leur angoisse devant la stasis, la crise politique généralisée. La loi est alors ce dont on ne discute pas, car si on en discutait, l'organisation même de la société exploserait. La loi est le sens, la crise le non sens. Mais dès lors, on ne peut faire évoluer la loi que de deux manières: ou bien on passe sous une autre loi, en se soumettant à un autre pouvoir; ou bien on change de pouvoir par une révolte ou une conjuration. Il n'y a pas d'ajustements progressifs de la loi. Il n'y a que des dégradations - peu à peu les lois ont de moins en moins de prise sur les citoyens - si bien que vient la crise, et le changement de régime, pour passer sous une autre loi.

Machiavel a tenté au contraire de penser une politique de la crise. Elle revient à situer le rôle du prince entre deux lois: la loi particulariste (celle des alliances entre grands, ou entre partis de la populace) et la loi qui reste un horizon, celle de l'unité de la république. Le prince est un briseur d'alliances, mais dans la visée de cette loi d'unification d'un peuple. La crise se met au service de cette loi. Mais une telle loi reste absente, voire utopique.

Inversement, l'utopie rousseauiste consiste à la fois à nous mettre devant la loi et à nous faire être la loi. La crise est toujours à l'horizon, mais elle vient de toutes les tentatives pour créer des partis qui brisent cette identité entre le sujet de la loi et le citoyen qui constitue avec tous ses pairs la volonté générale. C'est une utopie dangereuse, parce qu'elle exclut que la volonté générale puisse être tenue pour un compromis, et donc range dans le domaine de la crise partisane tous les ajustements et adaptations, qui obscurcissent forcément cette relation transparente du sujet au citoyen.

Les révolutionnaires (ainsi les marxistes) ont ensuite renversé le rapport des valeurs entre loi et crise. La loi n'était en fait qu'un paravent pour maintenir un pouvoir sans justice et empêcher la révolution nécessaire. La crise révolutionnaire est alors le seul moyen de renverser un système et d'aboutir à la justice sociale. Mais cette révolution n'offre ensuite ni législation ni ajustements possibles, seulement une perpétuelle révolution. Elle pose donc simplement un renversement de l'équation entre loi et sens. La loi devient le non sens, et la crise le sens. Mais une fois la crise passée, une fois la révolution accomplie, on reste confronté au non sens.

Sartre a été encore plus radical en pensant que le simple projet existentiel donnait du sens, mais que le sens se réduisait à chaque projet. Pour trouver un sens 
plus robuste et moins évanescent, il n'avait que le collectif. Mais un collectif sans loi peut-il se représenter à lui-même?

Les philosophies politiques contemporaines ont tenté au contraire de nous faire entrer dans l'espace de la loi, de nous faire nous y mouvoir, au lieu de rester devant la Loi. La proposition d'Habermas, mais aussi d'autres philosophes politiques (Dworkin et Rawls, de manières différentes) est de relier loi et discussion. Or toute discussion implique des échanges, des conversations, et donc des ajustements réciproques. On retrouve cela dans la notion d'équilibre réfléchi de Rawls, ou dans le processus de marché d'assurances imaginé par Dworkin comme pierre de touche de la justice sociale, ou encore dans son personnage du juge herculéen qui tente de rendre cohérente sa décision avec tous les principes qui inspiraient les jugements précédents, comme le ferait celui qui tenterait de résumer de manière cohérente les positions d'une conversation - Dworkin propose d'ailleurs plutôt une référence à la littérature qu'à la conversation, ce qui nous ramène à nos attracteurs fascinants. Le problème est que si nous prenons au sérieux le processus de la discussion, ou de la négociation, ou des échanges et ajustements, il est sans fin. Même sur la procédure de la discussion ou de la négociation, nous pourrons discuter. Les propositions de règles procédurales données par Rawls ou Dworkin restent elles-mêmes discutables. Or les ajustements réels et pratiques ne peuvent pas être sans fin. Il semble donc que Kafka ait eu raison: en tentant d'entrer dans l'enceinte de la Loi, nous continuons à adopter notre attitude de devant la Loi, qui nous donnait un rôle fini face à une entité surplombante capable d'emboîtements infinis.

Mais que ce soit en prônant la révolution, ou bien en reliant loi et discussion, il reste que, par rapport au paysan de Kafka, nous avons tenté d'entrer dans le château. Nous nous sommes dits que la porte était faite pour nous, et que ce serait notre cheminement de porte en porte qui ferait ce que nous sommes et ce que serait notre société. Dès lors, même si les philosophes conservent quelque peu l'attitude du paysan de Kafka, nous savons que la loi n'est pas surplombante, pas plus que la crise n'est forcément fatale à la société. Nous avons perdu notre crainte sacrée de la loi. Mais nous n'avons pas gagné de sens, parce que nous savons ce que vaut le sens que nous proposons: il ne vaut que ce que nous en avons fait. Nous avons alors des lois qui ne sont que le résultat temporaire de discussions et de négociations. Nous ne sommes plus devant la loi, mais entre les lois. Nous les faisons et nous jouons ensuite de leurs interstices pour profiter de leurs lacunes.

\section{LES AJUSTEMENTS ET LES LOIS D’AIRAIN}

Or maintenant que les lois servent seulement de cadres pour des ajustements, elles reviennent au niveau d'un rapport entre lois et ajustements qui avait commencé à trouver ses formes conceptuelles avec les réseaux financiers de la Renaissance, et qui de nos jours constitue notre référence sociale principale, à savoir la forme du marché. Jusqu'au milieu du XIX ${ }^{\mathrm{e}}$ siècle, on pouvait encore prétendre que le marché étant le domaine des ajustements que sont les marchandages, alors que le politique fixait un cadre de lois que tous les acteurs d'un Etat devaient considérer comme non négociables. Au marché les ajustements, au politique les lois. La situation s'est presque renversée. Le marché reste un réseau 
d'ajustements, mais pris globalement, ces ajustements obéissent à des lois d'airain, qui sont celles de l'économie. Il ne s'agit pas de lois que la nature impose à l'homme, mais de lois que par leurs activités d'échanges les humains s'imposent les uns les autres en produisant par leurs activités interindividuelles des effets collectifs qui retentissent sur tous. La valeur de la marchandise que j'offre est relative aux autres offres et aux demandes, si bien qu'elle ne peut résulter effectivement que d'ajustements. Mais personne ne maîtrise cette relativité, qui s'impose à tous. Nous sommes non pas devant, mais sous la Loi d'airain du marché, et cela même sur le plan théorique: si nous pouvons poser l'existence d'une solution au système des prix, nous ne savons pas comment déterminer constructivement cette solution. En particulier nous ne savons pas prédire les crises économiques, mais la régulation par crises rentre encore sous la loi de la relativité des échanges.

Inversement, le politique se charge maintenant essentiellement de régler les crises par des ajustements. Nous n'espérons plus résoudre les conflits. Mais nous avons maintenant en fait trois modes de traitement des conflits. Le premier consiste à leur donner des solutions partielles (par des discussions et négociations) qui les calment un moment puis les relancent. Le second est de les laisser en attente de règlements tant qu'ils ne bloquent pas nos coordinations sociales. C'est une mise en suspension. Le troisième est de faire reconnaître à l'avance que les tentatives de solution de certains conflits ne feront que les faire renaître, et que ce qui est alors stable, ce sont les pesanteurs qui empêchent ces conflits de se résoudre. On rentre alors sous le surplomb de la relativité économique et sociale.

\section{LES LOIS COMME EXPLORATIONS DES RELATIVITÉS SOCIALES}

En passant de devant la loi à sous la loi, nous nous sommes bornés à entrer dans la citadelle de la Loi, mais sans en modifier l'architecture. Or si nous abandonnons le fantasme de la Loi, nous pouvons penser les lois sous un autre mode. Il correspond plutôt à la conception de la démocratie comme démocratie délibérative que comme démocratie représentative ou participative. Il ne consiste ni à exprimer ce que nous voudrions que la volonté générale soit, et à assister ensuite à l'émergence transcendante de la volonté collective, ni à tenter des ajustements et des marchandages et à subir le retour des effets en réseau des échanges collectifs. Mais pour entrer dans ce nouveau mode il faut comprendre les lois comme institutions, et il faut avoir une idée plus précise et plus modeste à la fois de ce qu'est une institution.

Pour aller très vite, une institution prend appui sur l'existence préalable de pratiques d'interaction qui ont des effets collectifs. Mais ces effets comportent des aspects négatifs qu'il serait dans l'intérêt de la majorité de changer. Tous arrivent à imaginer d'autres interactions, qui pourraient produire des effets collectifs moins gênants. Mais tous voient aussi que tant que ces interactions ne seront pas le fait de la majorité, les effets collectifs pourraient être pires, et que quand elles le seront, il sera possible à certains de profiter de l'effort collectif majoritaire. Il n'est donc pas possible de commencer chacun dans son coin à lancer ces interactions souhaitables. Il faut le faire en commun, et en acceptant l'existence de profiteurs. Cela suppose donc que la majorité envisage de changer ses motivations - 
puisque les motivations actuelles conduiraient rationnellement chaque individu séparément à se résigner à la situation actuelle: ou bien il risque une situation pire, ou il a intérêt à jouer le rôle du profiteur. Cette majorité se propose de se donner les motivations qu'elle aura si une majorité future choisit ces modes d'interactions: poursuivre la coopération et utiliser certains de ses bénéfices pour sanctionner une partie des profiteurs. Il deviendra alors rationnel de coopérer. Une fois que la majorité s'est convaincue que si la coopération avait lieu, les motivations qui la justifient seraient stables et efficaces, et une fois qu'elle a pu admettre que la situation future, évaluée selon ses motivations actuelles - mais possible seulement par un changement de motivations - serait préférable, cette majorité prend le risque d'agir dans le présent comme si elle avait déjà ces motivations futures. Pour donner des repères à tous, on fixe donc des règles de cette coopération. Et si tout cela fonctionne, nous avons une institution. Les lois alors sont simplement les règles nécessaires au bon fonctionnement des institutions.

Les institutions, et donc les lois, sont des moyens par lesquels une société se hisse au dessus des interactions qui tissent le réseau de relativités qui la constituent, et tente de passer à un autre réseau de relativités. L'économie actuelle ne permet pas de penser les institutions, mais il ne faut pas désespérer. La difficulté théorique est que le problème d'évaluer ce que donne de manière collective un changement de motivation, qui change en fait la situation et le réseau de relativités, est un problème complexe. En pratique, nous ne savons pas à l'avance dans quelle mesure une institution projetée va fonctionner comme prévu. La création institutionnelle va toujours un peu à l'aventure.

Imaginons un paysage de crêtes, qui sépareraient des bassins, qui représenteraient les formes de sociétés qui s'auto-entretiennent de concert avec leur environnement. Certains bassins sont plus larges et plus profonds, et plus attractifs. Les institutions sont constituées pour nous permettre de passer d'un bassin moins profond et moins satisfaisant à un bassin plus satisfaisant. Mais quand nous faisons l'effort de franchir le col, nous ne savons pas si le bassin suivant tiendra ses promesses, parce que l'activité institutionnelle change le paysage, et donc la configuration des bassins et des crêtes, d'une manière que nous sommes incapables de prévoir de manière précise et assurée.

Les lois sont alors des frontières et des poteaux indicateurs, qui permettent à nos activités de réaliser les institutions. Elles ont donc à la fois une fonction régulatrice et une fonction exploratrice. Les sociétés, par leurs lois, explorent les mondes des sociétés possibles. Nous ne sommes donc plus devant la Loi. Nous utilisons les lois comme des boussoles, comme des rambardes, mais aussi comme des outils pour refaçonner le paysage social.

Mais si les lois sont devenues instrumentales, la perte de leur aura sacrée ne va-t-elle pas affaiblir voir annuler leur puissance et leur efficace? François Ost s'est en particulier inquiété de la durée de plus en plus brève de la mise en œuvre des lois. Si les lois sont des instruments, on peut les modifier sans cesse. Elles sont soumises à ajustements. Mais que valent des repères que l'on déplace sans arrêt? Ce problème ne peut être contourné que si la formulation des lois est suffisamment vague pour qu'elles puissent s'appliquer même quand les circonstances ont un peu changé. Elles continuent à jouer alors le rôle de repères, et les ajustements sont faits par l'évolution des jugements qui les invoquent cas par cas. C'était déjà ainsi que fonctionnait le système juridique, mais à présent ce 
rôle d'ajustement des jugements est affiché, alors qu'il était dissimulé par le passé.

Or en utilisant les lois comme repères dans notre exploration institutionnelle des sociétés possibles, nous allons aussi mettre au jour ce qui reste non dit mais présent en arrière-plan dans l'histoire de Kafka: la motivation de l'homme de la campagne, ce que certains ont appelé le désir de la Loi, un désir très fort, puisqu'il passe sa vie rongé par ce désir et qu'il finit par en mourir. En effet constituer une institution, c'est changer nos motivations. Mais en tentant de les changer nous découvrons aussi celles de nos motivations qui résistent à ces changements. Quand nous tentons de généraliser l'institution du marché, qui repose sur des motivations qui sont liées à la concurrence, donc sur la vanité d'avoir fait une meilleure affaire que les autres, nous nous apercevons que d'autres motivations, comme le désir d'être reconnu de manière durable par ceux qui ont un statut semblable au nôtre, rentrent en conflit avec cette motivation de la concurrence, et qu'elles persistent à rendre angoissante la vie de celui qui raisonne seulement en termes de concurrence. Notre exploration nous fait donc découvrir deux sortes de contraintes, qui ne sont plus des lois d'airain, mais des forces de rappel. Les premières, ce sont les contraintes propres au fonctionnement des institutions. Les lois ne déterminent pas directement les activités, elles leur servent de repères, et donnent donc lieu à d'autres activités possibles grâce à ces repérages. Le système des activités ainsi créées peut s'auto-maintenir ou bien dégénérer, et nous découvrons alors les contraintes de ces interrelations. Les secondes, ce sont les contraintes du réseau de nos propres motivations. En changeant certaines de ces motivations, nous nous opposons à la résistance de certaines autres dont l'importance nous est ainsi révélée, ou encore nous découvrons que la conjugaison de différentes motivations mène à des emballements enthousiasmants ou au contraire effroyables (comme ceux qui mènent aux génocides). Ce ne sont plus les lois qui nous dominent alors, mais les contraintes de nos pulsions ainsi que nos interactions collectives qui nous sont révélées. Les lois y ont perdu en sacralisation, mais elles y ont gagné en reconnaissance, car c'est à elles que nous devons toujours les institutions qui sont nos moyens d'explorer nos possibilités sociales.

Université de Provence 